Sugar - sweet ened bever age and di et soda consumption and the 7-year risk for type 2 di abet es mel litus in midl e- aged Japanese men

\begin{tabular}{|l|l|}
\hline 著者 & $\begin{array}{l}\text { Sakur ai Nasar u, Nakamur a Koshi, M ur a } \\
\text { Kat suyuki, Takamur a Toshi nar i, Yoshi ta } \\
\text { Kat sushi, Nagasawa Shi n- ya, Nor i kawa Yuko, } \\
\text { I shi zaki Nasao, K do Ter uhi ko, Nar use Yuchi, } \\
\text { Suwazono Yasushi, Sasaki Sat oshi, Nakagawa } \\
\text { Hi deaki }\end{array}$ \\
\hline $\begin{array}{l}\text { j our nal or } \\
\text { publ i cat i on ti t l e }\end{array}$ & Eur opean Jour nal of Nut ri ti on \\
\hline vol une & 53 \\
\hline number & 1 \\
\hline page r ange & $251-258$ \\
\hline year & 2014 02- 01 \\
\hline URL & ht t p: //hdl . handl e. net /2297/34671 \\
\hline
\end{tabular}




\title{
Sugar-sweetened beverage and diet soda consumption and the 7-year risk for type 2 diabetes mellitus in middle-aged Japanese men
}

\author{
M. Sakurai $\cdot$ K. Nakamura $\cdot$ K. Miura $\cdot$ T. Takamura $\cdot$ K. Yoshita $\cdot$ S.Y. Nagasawa $\cdot$ Y. Morikawa $\cdot$ M. Ishizaki $\cdot$ \\ T. Kido $\cdot$ Y. Naruse $\cdot$ Y. Suwazono $\cdot$ S. Sasaki $\cdot$ H. Nakagawa \\ Masaru Sakurai \\ Department of Epidemiology and Public Health, Kanazawa Medical University, Ishikawa, Japan, and \\ Department of Preventive Medicine, Northwestern University Feinberg School of Medicine, Chicago, IL, USA \\ K. Nakamura $\cdot$ S.Y. Nagasawa $\cdot$ Y. Morikawa $\cdot$ H Nakagawa \\ Department of Epidemiology and Public Health, Kanazawa Medical University, Ishikawa, Japan
}

K. Miura

Department of Health Science, Shiga University of Medical Science, Otsu, Japan

\section{T. Takamura}

Department of Disease Control and Homeostasis, Kanazawa University Graduate School of Medical Science, Kanazawa, Japan

K. Yoshita

Department of Food Science and Nutrition, Graduate School of Human Life Science, Osaka City University, Osaka, Japan

M. Ishizaki

Department of Social and Environmental Medicine, Kanazawa Medical University, Ishikawa, Japan

\section{T. Kido}

School of Health Sciences, College of Medical, Pharmaceutical and Health Sciences, Kanazawa University, Kanazawa, Japan

Y. Naruse 
Department of Community and Geriatric Nursing, Toyama University, Toyama, Japan

\author{
Y. Suwazono \\ Department of Occupation and Environmental Medicine, Graduate School of Medicine, Chiba University, \\ Chiba, Japan
}

\title{
S. Sasaki
}

Department of Social and Preventive Epidemiology, School of Public Health, the University of Tokyo, Tokyo, Japan

\section{Correspondence to:}

Masaru Sakurai, Department of Epidemiology and Public Health, Kanazawa Medical University, 1-1 Daigaku, Uchinada, Ishikawa 920-0293, Japan.

Fax: +81-76-286-3728; Tel: +81-76-286-2211;

E-mail: m-sakura@kanazawa-med.ac.jp 


\begin{abstract}
Purpose This cohort study investigated the association between sugar-sweetened beverage (SSB) and diet soda consumption and the incidence of type 2 diabetes in Japanese men.

Methods The participants were 2,037 employees of a factory in Japan. We measured consumption of SSB and diet soda using a self-administered diet history questionnaire. The incidence of diabetes was determined in annual medical examinations over a 7-year period. Hazard ratios (HRs) with 95\% confidence intervals (CIs) for diabetes were estimated after adjusting for age, BMI, family history, and dietary and other lifestyle factors.

Results During the study, 170 participants developed diabetes. The crude incidence rates (/1000 person-years) across participants who were rare/never SSB consumers, $<1$ serving/week, $\geq 1$ serving/week and $<1$ serving/day, and $\geq 1$ serving/day were $15.5,12.7,14.9$, and 17.4, respectively. The multivariate adjusted HR compared to rare/never SSB consumers was 1.35 (95\% CI, 0.80-2.27) for participants who consumed $\geq 1$ serving/day SSB. Diet soda consumption was significantly associated with the incident risk of diabetes ( $P$ for trend $=0.013$ ), and multivariate-adjusted HRs compared to rare/never diet soda consumers were 1.05 (0.62-1.78) and 1.70 (1.13-2.55), respectively, for participants who consumed $<1$ serving/week and $\geq 1$ serving/week.

Conclusions Consumption of diet soda was significantly associated with an increased risk for diabetes in Japanese men. Diet soda is not always effective at preventing type 2 diabetes even though it is a zero-calorie drink.
\end{abstract}

\title{
Key words
}

cohort study, epidemiology, incidence, nutrition 


\section{Introduction}

The increasing number of patients with diabetes in Asian countries is an important public health problem [1]. One well-documented change that may contribute to the risk of diabetes in Asia and elsewhere is soft drink consumption [2-4]. In fact, trends of increased soft drink consumption have been observed not only in Western but also in Asian countries [5].

In many previous studies and meta-analyses [6-12], consumption of soft drinks and sugar-sweetened beverages (SSB) has been associated with the incidence of type 2 diabetes. These studies suggested that the effects of soft drinks on type 2 diabetes are partly mediated by body mass index (BMI) [6, 10, 12-14]. However, these studies were performed mainly in Western countries [6-9, 11-15], and it is unclear whether similar associations between soft drink consumption and the risk of diabetes would be observed in relatively lean Asian people.

Only two previous studies have evaluated the association between diet soda intake and the incidence of type 2 diabetes $[11,15]$. While one previous study indicated a significant association even after adjustment for BMI and other lifestyle-related factors [15], the other did not [11], and it remains unclear whether diet soda consumption is associated with the incident risk of diabetes mellitus.

In this cohort study, we examined the associations of SSB and diet soda consumption with the 7-year incidence of type 2 diabetes mellitus in relatively lean middle-aged Japanese men.

\section{Participants and Methods}

\section{Participants}

The study participants were employees of a factory that produces zippers and aluminum sashes in Toyama Prefecture, Japan. Detailed information on the study population has been reported previously [16-19]. The Industrial Safety and Health Law in Japan requires that employers provide annual health examinations for all employees. A test for diabetes mellitus was conducted during annual medical examinations between 2003 and 2010. In 2003, 2,275 (89\%) of 2,543 male employees aged 35-55 years received health examinations and responded to the diet survey. Of these 2,275 potential participants, 238 (10\%) were excluded for the following reasons: 165 had diabetes or high levels of fasting plasma glucose ( $\geq 126 \mathrm{mg} / \mathrm{dL}$ ) or glycated hemoglobin (HbA1c) ( $\geq 6.5 \%)$ at the time of the baseline examination; 13 had a total daily energy intake of below $500 \mathrm{kcal}$ or above 5,000 kcal; soft drink consumption data were unavailable for 13; and 47 did not participate in consecutive annual follow-up health examinations. The remaining 2,037 participants were included in the present study. 


\section{Baseline Examination}

The annual health examination included medical history, physical examination, anthropometric measurements, and measurement of fasting plasma glucose, HbA1c, and serum lipid levels. Height was measured without shoes to the nearest $0.1 \mathrm{~cm}$ using a stadiometer. Weight was measured with participants wearing only light clothing and no shoes to the nearest $0.1 \mathrm{~kg}$ using a standard scale. BMI was calculated as weight/height ${ }^{2}\left(\mathrm{~kg} / \mathrm{m}^{2}\right)$. Blood pressure was measured using an automatic manometer (BP 103i; Nippon Colin, Komaki, Japan) after the subject had rested for 5 min in a seated position. All measurements were taken by trained staff.

Plasma glucose levels were measured enzymatically by glucose UV test (Abbott Laboratories, Chicago, IL), and plasma insulin levels were determined by radioimmunoassay (Shionogi Co., Tokyo, Japan). HbA1c was measured by high-velocity liquid chromatography using a fully automated hemoglobin A1c analyzer (Kyoto Daiichi Kagaku, Kyoto, Japan). Quality control of the HbA1c measurements was performed using the standard certified by the Japan Diabetes Society (JDS), and HbA1c values were converted to National Glycohemoglobin Standardization Program (NGSP) values using the formula provided by the JDS: HbA1c (NGSP) $=$ HbA1c (JDS) +0.4 [20]. All of the analyses reported here adopted the HbA1c values obtained by the NGSP method. Total cholesterol and triglycerides were measured by enzymatic assay. HDL-cholesterol was measured using direct methods. Insulin resistance (IR) was calculated by the homeostasis model assessment (HOMA) method using the formula: HOMA-IR=fasting insulin $(\mu \mathrm{U} / \mathrm{mL}) \times$ fasting plasma glucose $(\mathrm{mg} / \mathrm{dL}) / 405[21]$.

A questionnaire was used to collect information about smoking, alcohol consumption, habitual exercise, family history of diabetes, medical history of hypertension, dyslipidemia, diabetes, and the use of antidiabetic medication. Soft drink consumption, total energy intake (kcal/day), and dietary fiber intake (g/day) were assessed using a self-administered diet history questionnaire (DHQ) [22]. The DHQ was developed for epidemiological studies in Japan to estimate the dietary intakes of macronutrients and micronutrients during the previous month. A detailed description of the methods used to calculate dietary intakes and the validity of the DHQ have been reported previously [22-25]. In the DHQ, the consumption of beverages, such as "non-calorie carbonated soft drinks" (hereafter referred to as “diet soda”), "regular soft drinks, sugar-sweetened soda, and sports drinks, excluding 100\% fruit juice and vegetable juice” (hereafter referred to as “SSB”), “100\% fruit juice,” “vegetable juice,” and “coffee,” were evaluated. Frequency response options for these items were as follows: rare/never, 1/week, 2-3/week, 4-6/week, 1/day, 2-3/day, 4-5/day, or 6+/day. Participants reported serving sizes in five categories: $<50 \%, 70-80 \%, 100 \%$, $120-130 \%$, and $>150 \%$ of one glass $(250 \mathrm{~mL}$ ) for soft drinks and one cup (150 mL) for coffee. To allow comparison 
with previous studies, estimated soft drink consumption was converted from $\mathrm{mL}$ into servings with one serving assigned a value of $237 \mathrm{~mL}$ or $8 \mathrm{oz}$. Consumption of SSB and diet soda was categorized as rare/never, more often than rare/never but $<1$ serving/week, $\geq 1$ serving/week but $<1$ serving/day, and $\geq 1$ serving/day. As the number of participants who consumed $\geq 1$ serving of diet soda per day was small $(n=20)$, we also used the following three categories of diet soda consumption: rare/never, more often than rare/never but $<1$ serving/week, and $\geq 1$ serving/week. In a previous validation study among 47 women, the Pearson correlation coefficient between DHQ and 3-day estimated dietary records was 0.48 for energy [22]. The Pearson correlation coefficients between DHQ and 16-day weighed dietary record among 92 women were 0.28 for energy and 0.69 for dietary fiber, and the Spearman correlation coefficient was 0.55 for soft drinks (Sasaki S, unpublished observations, 2007).

\section{Diagnosis of diabetes}

Fasting plasma glucose and HbA1c were measured during the annual medical examinations. According to the definition of the American Diabetes Association [26] and the JDS [20], the diagnosis of diabetes was confirmed by at least one of the following observations: 1) a fasting plasma glucose concentration $\geq 126 \mathrm{mg} / \mathrm{dL}$; 2) HbA1c value $\geq 6.5 \%$; and 3) treatment with insulin or oral hypoglycemic agent.

\section{Statistical analysis}

Mean baseline values of BMI, blood pressure, plasma glucose, and serum lipids were determined for each category of soft drink consumption. Statistical analyses were performed on triglycerides, fasting insulin, and HOMA-IR values transformed on a log scale. We calculated crude incidence rates and hazard ratios (HRs) for diabetes according to the categories of SSB and diet soda consumption. The Cox proportional hazard model was used to calculate HRs. Adjustment for possible confounders was performed sequentially as follows: for age (Model 1), for age and BMI (Model 2); adjustment also for family history of diabetes (no, yes), smoking status (never smoker, ex-smoker or current smoker), alcohol consumption (nondrinker, occasional drinker, consumption $<20 \mathrm{~g}$ /day, consumption $\geq 20 \mathrm{~g} /$ day), habitual exercise (no, yes), presence of hypertension (no, yes), presence of dyslipidemia (no, yes), dietary intervention for chronic disease (no, yes), total energy intake (kcal/day), and dietary fiber intake (g/day) (Model 3); and adjustment also for consumption of SSB (diet soda), diet soda (SSB), fruit juice, vegetable juice, and coffee (Model 4). Statistical analyses were conducted using the Japanese version of the Statistical Package for the Social Sciences (SPSS version 17.0; Tokyo, Japan). In all analyses, $P<0.05$ was considered statistically significant.

\section{Ethical considerations}


Written informed consent was not obtained from the participants. The design of the present study was approved by the occupational safety and health committee of the subject company, which consisted of employee representatives. Employees were informed of the study design and of the right to refuse to participate in the study by the documents. Participants who answered the questionnaire were regarded as having consented to the survey. Linkable anonymized data were provided by the company to ensure that individuals would not be identifiable by the researchers. The present study was approved by the Institutional Review Committee of Kanazawa Medical University for Ethical Issues.

\section{Results}

The mean age of the participants at baseline was 46.2 years and mean BMI was $23.4 \mathrm{~kg} / \mathrm{m}^{2}$. Median (range) SSB consumption was $0.2(0.0$ - 9.6) servings/day and median (range) diet soda consumption was 0.0 (0.0-4.7) servings/day. Among the soft drinks examined, modest but significant associations were observed between consumption of SSB and diet soda ( $r=0.284, P<0.001)$, between SSB and $100 \%$ fruit juice $(r=0.295, P<0.001)$, and between diet soda and $100 \%$ fruit juice $(r=0.166, P<0.001)$. Vegetable juice consumption and coffee consumption were not associated with consumption of other soft drinks.

At the baseline examination, those who consumed larger amounts of SSB were significantly younger and had higher values for BMI, fasting insulin, HOMA-IR, and total energy intake, lower serum HDL-cholesterol levels, and lower values for energy-adjusted dietary fiber intake (Table 1). Those who consumed larger amounts of diet soda were significantly younger, and had higher values for BMI and total energy intake (Table 2).

During the 7-year follow up (11,253 person-years, mean follow-up time $5.5 \pm 1.8$ years), we documented 170 cases of diabetes, of which 79 were diagnosed based on high fasting plasma glucose levels, 73 were based on high HbA1c levels, and 18 were based on both high fasting plasma glucose and high HbA1c levels.

The crude incidence rates (per 1,000 person-years) across the SSB consumption categories from lowest to highest were 15.5, 12.7, 14.9, and 17.4, respectively (Table 3). Multivariate-adjusted HRs (95\%CI) across SSB consumption from lowest to highest were 1.00 (reference), 0.90 (0.80-2.27), 1.06 (0.74-1.53), and 1.35 (0.80-2.27), respectively, and no significant association between SSB consumption and the incidence of diabetes was observed.

The crude incidence rates across the diet soda consumption categories from lowest to highest were 13.8, 15.0, 25.4, 
and 17.2, respectively (Table 3). The age-adjusted HR for participants who consumed at least one serving of diet soda per week was significantly higher than that for those who did not consume diet soda. Similar associations were found after adjustment for BMI (Model 2), other potential confounders (Model 3), and the consumption of other soft drinks (Model 4). The results were similar even when participants who had received a dietary intervention for hypertension, hyperlipidemia and other chronic disease from their physicians, nurses, and dietitians were excluded from the analyses $(n=1,724$, data not shown).

\section{Discussion}

We investigated the association between consumption of soft drinks and the incidence of type 2 diabetes in middle-aged Japanese men. SSB consumption was not associated with incidence of diabetes. Diet soda consumption was significantly associated with the incidence of diabetes, and participants who consumed at least one diet soda serving per week had about a 70\% greater risk of diabetes compared to those who did not consume diet soda.

Only two previous studies have evaluated the association between diet soda intake and the incidence of type 2 diabetes [11, 15]. The results from Multi-Ethnic Study of Atherosclerosis (MESA) showed a significant association even after adjustment for BMI and other lifestyle-related factors [15], and adjusted HRs for participants who consume diet soda $\geq 1$ serving/week to $<1$ /serving/day and for who consume $\geq 1$ serving/day were 1.23 (95\%CI 0.94-1.60) and 1.40 (1.06-1.84), respectively. In the Health Professionals Follow-Up Study [11], artificially sweetened beverages were significantly associated with type 2 diabetes in the age-adjusted analysis. However, participants who consumed artificially sweetened beverages were more likely to have reported either weight gain or weight loss before the start of the study, to have tried a low-calorie diet, and to have factors associated with a risk of diabetes such as family history of diabetes, prevalence of hypertension and use of diuretics, and consumption of artificially sweetened beverages was not associated with diabetes after adjustment for these confounders. In our study participants, diet soda consumption was associated with not only BMI and total energy intake, but also with lifestyle factors associated with the risk of diabetes, such as higher SSB consumption, lower fiber intake. Further, consumption tended to be associated with a higher percentage of participants who had received dietary intervention for hypertension, hyperlipidemia, and other chronic diseases. However, a significant positive association between diet soda consumption and incidence of diabetes was found even after adjustment for these factors. These results suggest that diet soda consumption itself may increase the risk of diabetes.

In many previous studies and meta-analyses [6-12], SSB consumption was shown to be associated with the incidence 
of type 2 diabetes. However, SSB consumption was not associated with the incidence of diabetes in the present study. The Atherosclerosis Risk in Communities Study also failed to show a consistent association between SSB intake and incident type 2 diabetes [13]. As discussed by the authors, their participants were older (mean age 53.6 years) and heavier (mean BMI $27.2 \mathrm{~kg} / \mathrm{m}^{2}$ ) compared to those in other similar studies, which may have affected the results. Our study participants were younger and less obese, and median SSB consumption was as low as 0.20 servings (1.6 oz)/day; only $12 \%$ of participants consumed $\geq 1$ serving of SSB per day. Furthermore, the HR of participants who consumed one serving or more of SSB was 1.20-1.34; these values were similar to recent meta-analyses that compared the risk of type 2 diabetes between extreme quantiles of SSB intake [12]. Estimated sample sizes to detect the significant difference using these data of previous studies was about 13,000 parson-years in each category of SSB consumption. Therefore, the lower number of participants, particularly of SSB consumers, in our study may have affected the results.

SSB are thought to lead to weight gain $[6,8]$. Since the effect of SSB on type 2 diabetes is partly mediated by BMI, the association between SSB and the incidence of diabetes was diminished or even not significant after adjustment for BMI in previous studies [6, 10, 12-14]. In the present study, SSB consumption was significantly associated with BMI at the baseline examination. Similar to SSB, an association between diet soda consumption and subsequent weight gain is plausible. Artificial sweeteners contained in diet soda may increase desire for sweetness and more energy-dense foods [27-29]. Overestimation of the number of calories saved by substituting diet soda for SSB may cause overconsumption of other foods/beverages [30]. In our study, diet soda consumption was associated with baseline BMI and total energy intake. Furthermore, the association between diet soda intake and weight gain may be biased by early awareness of energy imbalance, i.e., diet soda consumption may serve as an early attempt to maintain weight. There are many confounding factors on the association between diet soda consumption, body weight gain, and the incidence of diabetes, and further study is needed to evaluate the effect of diet soda itself on future body weight gain and the incidence of diabetes.

Diet soda and some artificial sweeteners have been reported to affect incretin secretion [31-34], which may affect glucose/insulin metabolism. However, artificial sweeteners and diet soda increased GLP-1 secretion in subjects with type 1 diabetes and healthy control subjects, but not in subjects with type 2 diabetes [31, 32]. Although diet soda is non-caloric and increases incretin secretion, consumption of diet soda to prevent or treat diabetes would be ineffective.

The strength of this study was that this is the first study to evaluate the relationship between diet soda intake and the 
incidence of diabetes in an Asian population. Moreover, several previous cohort studies used information on incident diabetes collected from self-administered questionnaires, whereas our conclusions were based on more reliable data obtained from annual examinations and fasting blood glucose and HbA1c. There were some limitations to this study. First, the lower number of participants may have affected the negative association between SSB consumption and the incidence of diabetes in this study. Second, the consumption of soft drinks and other lifestyle factors associated with the risk of diabetes were evaluated only at the baseline examination; changes during the 7-year follow-up period were not considered. Third, the sample included only males who were employed. Poor health may exclude some individuals from working and therefore the prevalence of obesity or incidence of diabetes may have been lower in our sample than in the general Japanese population. Fourth, we did not determine whether the diabetes that developed was type 1 or type 2 . However, the study participants were middle-aged men, and as the condition was detected in an annual medical check-up, with relatively mild diabetes being found, it is likely that the cases were of type 2 diabetes.

In conclusion, diet soda was associated with a significantly increased risk of diabetes in middle-aged Japanese men. Our results indicate that diet soda is not always effective at preventing type 2 diabetes even though it is a zero-calorie drink.

\section{Acknowledgements}

This research was supported by a Grant-in-Aid from the Ministry of Health, Labor, and Welfare, Health and Labor Sciences research grants, Japan (H18-Junkankitou[Seishuu]-Ippan-012, H19-Junkankitou [Seishuu]-Ippan-012, H19Junkankitou [Seishuu]-Ippan- 021, H20-Junkankitou [Seishuu]-Ippan-013, H22- Junkankitou [Seishuu]-Ippan-005, H23-Junkankitou [Seishuu]-Ippan-005); a Grant-in-Aid from the Ministry of Education, Culture, Sports, Science and Technology of Japan for Scientific Research (B) (20390188); and the Japan Arteriosclerosis Prevention Fund.

\section{Conflict of interest}

The authors declare that they have no conflict of interest.

The English in this document has been checked by at least two professional editors, both native speakers of English. For a certificate, please see: 
http://www.textcheck.com/certificate/mt4aNz 


\section{References}

1. Yoon KH, Lee JH, Kim JW, Cho JH, Choi YH, Ko SH, et al (2006) Epidemic obesity and type 2 diabetes in Asia. Lancet 368:1681-1688

2. Bray GA, Nielsen SJ, Popkin BM (2004) Consumption of high-fructose corn syrup in beverages may play a role in the epidemic of obesity. Am J Clin Nutr 79:537-543

3. Ismail AI, Tanzer JM, Dingle JL (1997) Current trends of sugar consumption in developing societies. Community Dent Oral Epidemiol 25:438-443

4. Basu M (2007) Diabetes, obesity and soft drinks. Natl Med J India 20:102-103

5. Zenith international’s report on global soft drinks (2008) Bath, United Kingdom: Zenith International, Ltd.

6. Schulze MB, Manson JE, Ludwig DS, Colditz GA, Stampfer MJ, Willett WC, et al (2004) Sugar-sweetened beverages, weight gain, and incidence of type 2 diabetes in young and middle-aged women. JAMA 292:927-934

7. Dhingra R, Sullivan L, Jacques PF, Wang TJ, Fox CS, Meigs JB, et al (2007) Soft drink consumption and risk of developing cardiometabolic risk factors and the metabolic syndrome in middle-aged adults in the community. Circulation 116:480-488

8. Palmer JR, Boggs DA, Krishnan S, Hu FB, Singer M, Rosenberg L (2008) Sugar-sweetened beverages and incidence of type 2 diabetes mellitus in African American women. Arch Intern Med 168:1487-1492

9. Bazzano LA, Li TY, Joshipura KJ, Hu FB (2008) Intake of fruit, vegetables, and fruit juices and risk of diabetes in women. Diabetes Care 31:1311-1317

10. Odegaard AO, Koh WP, Arakawa K, Yu MC, Pereira MA (2010) Soft drink and juice consumption and risk of physician-diagnosed incident type 2 diabetes: the Singapore Chinese Health Study. Am J Epidemiol 171:701-708

11. de Koning L, Malik VS, Rimm EB, Willett WC, Hu FB (2011) Sugar-sweetened and artificially sweetened beverage consumption and risk of type 2 diabetes in men. Am J Clin Nutr 93:1321-1327

12. Malik VS, Popkin BM, Bray GA, Despres JP, Willett WC, Hu FB (2010) Sugar-sweetened beverages and risk of metabolic syndrome and type 2 diabetes: a meta-analysis. Diabetes Care 33:2477-2483

13. Paynter NP, Yeh HC, Voutilainen S, Schmidt MI, Heiss G, Folsom AR, et al (2006) Coffee and sweetened beverage consumption and the risk of type 2 diabetes mellitus: the atherosclerosis risk in communities study. Am J Epidemiol 164:1075-1084

14. Montonen J, Jarvinen R, Knekt P, Heliovaara M, Reunanen A (2007) Consumption of sweetened beverages and intakes of fructose and glucose predict type 2 diabetes occurrence. J Nutr 137:1447-1454

15. Nettleton JA, Lutsey PL, Wang Y, Lima JA, Michos ED, Jacobs DR Jr. (2009) Diet soda intake and risk of incident metabolic syndrome and type 2 diabetes in the Multi-Ethnic Study of Atherosclerosis (MESA).Diabetes Care 32:688-694 
16. Sakurai M, Miura K, Takamura T, Ishizaki M, Morikawa Y, Nakamura K, et al (2009) J-shaped relationship between waist circumference and subsequent risk for Type 2 diabetes: an 8-year follow-up of relatively lean Japanese individuals. Diabet Med 26:753-759.

17. Nakashima M, Sakurai M, Nakamura K, Miura K, Yoshita K, Morikawa Y, et al (2010) Dietary glycemic index, glycemic load and blood lipid levels in middle-aged Japanese men and women. J Atheroscler Thromb $17: 1082-1095$

18. Sakurai M, Nakamura K, Miura K, Takamura T, Yoshita K, Nagasawa SY, et al (2012) Dietary glycemic index and risk of type 2 diabetes in middle-aged Japanese men. Metabolism 61:47-55

19. Sakurai M, Nakamura K, Miura K, Takamura T, Yoshita K, Nagasawa SY, et al (2012) Self-reported speed of eating and 7-year risk of type 2 diabetes mellitus in middle-aged Japanese men. Metabolism. 61:1566-1571

20. The Committee of the Japan Diabetes Society on the diagnostic criteria of diabetes mellitus (2012) Report of the Committee on the classification and diagnostic criteria of diabetes mellitus. J Diabetes Invest 3:39-40

21. Matthews DR, Hosker JP, Rudenski AS, Naylor BA, Treacher DF, Turner RC (1985) Homeostasis model assessment: insulin resistance and beta-cell function from fasting plasma glucose and insulin concentrations in man. Diabetologia 28:412-419

22. Sasaki S, Yanagibori R, Amano K (1998) Self-administered diet history questionnaire developed for health education: a relative validation of the test-version by comparison with 3-day diet record in women. $\mathrm{J}$ Epidemiol 8:203-215

23. Sasaki S, Ushio F, Amano K, Morihara M, Todoriki O, Uehara Y, et al (2000) Serum biomarker-based validation of a self- administered diet history questionnaire for Japanese subjects. J Nutr Sci Vitaminol 46:285-296

24. Okubo H, Sasaki S, Rafamantanantsoa HH, Ishikawa-Takata K, Okazaki H, Tabata I (2008) Validation of self-reported energy intake by a self-administered diet history questionnaire using the doubly labeled water method in 140 Japanese adults. Eur J Clin Nutr 62:1343-1350

25. Yamada M, Murakami K, Sasaki S, Takahashi Y, Okubo H (2008) Soft drink intake is associated with diet quality even among young Japanese women with low soft drink intake. J Am Diet Assoc 108:1997-2004

26. American Diabetes Association (2012) Diagnosis and Classification of Diabetes Mellitus. Diabetes Care 35:S64-S71

27. Blundell JE, Hill AJ (1986) Paradoxical effects of an intense sweetener (aspartame) on appetite. Lancet 1:1092-1093

28. Rogers PJ, Blundell JE (1989) Separating the actions of sweetness and calories: effects of saccharin and carbohydrates on hunger and food intake in human subjects. Physiol Behav 45:1093-1099

29. Tordoff MG, Alleva AM (1990) Oral stimulation with aspartame increases hunger. Physiol Behav 47:555-559 
30. Swithers SE, Davidson TL (2008) A role for sweet taste: calorie predictive relations in energy regulation by rats. Behav Neurosci 122:161-173

31. Brown RJ, Walter M, Rother KI (2012) Effects of diet soda on gut hormones in youths with diabetes. Diabetes Care 35:959-964

32. Brown RJ, Walter M, Rother KI (2009) Ingestion of diet soda before a glucose load augments glucagon-like peptide-1 secretion. Diabetes Care 32:2184-2186

33. Wu T, Zhao BR, Bound MJ, Checklin HL, Bellon M, Little TJ, et al (2012) Effects of different sweet preloads on incretin hormone secretion, gastric emptying, and postprandial glycemia in healthy humans. Am J Clin Nutr 95:78-83

34. Ma J, Bellon M, Wishart JM, Young R, Blackshaw LA, Jones KL, et al (2009) Effect of the artificial sweetener, sucralose, on gastric emptying and incretin hormone release in healthy subjects. Am J Physiol Gastrointest Liver Physiol 296:G735-G739 
Table 1. Baseline characteristics of the 2,037 participants according to sugar-sweetened beverage consumption category

\begin{tabular}{|c|c|c|c|c|c|c|c|c|c|c|c|c|c|}
\hline \multirow[b]{3}{*}{$\mathrm{N}$} & \multicolumn{13}{|c|}{ Sugar-sweetened beverage consumption } \\
\hline & \multicolumn{3}{|c|}{ Rare or never } & \multicolumn{3}{|c|}{$\begin{array}{c}\text { > rare/never } \\
\text { but }<1 \text { serving/week }\end{array}$} & \multicolumn{3}{|c|}{$\begin{array}{l}\geq 1 \text { serving/week } \\
\text { to }<1 \text { serving/day }\end{array}$} & \multicolumn{3}{|c|}{$\geq 1$ serving/day } & \multirow[t]{2}{*}{$\mathrm{p}^{\mathrm{b}}$} \\
\hline & 660 & & & 271 & & & 865 & & & 241 & & & \\
\hline Age (years) & 47.0 & \pm & 6.1 & 46.2 & \pm & 6.3 & 45.9 & \pm & 5.8 & 45.2 & \pm & 6.1 & 0.001 \\
\hline BMI $\left(\mathrm{kg} / \mathrm{m}^{2}\right)$ & 23.2 & \pm & 2.9 & 22.9 & \pm & 2.6 & 23.5 & \pm & 2.9 & 23.7 & \pm & 3.1 & 0.013 \\
\hline Systolic blood pressure (mmHg) & 121.4 & \pm & 17.6 & 119.0 & \pm & 18.8 & 120.3 & \pm & 19.0 & 120.2 & \pm & 16.5 & 0.659 \\
\hline Diastolic blood pressure (mmHg) & 78.1 & \pm & 12.5 & 76.5 & \pm & 13.3 & 77.7 & \pm & 13.5 & 78.0 & \pm & 11.7 & 0.799 \\
\hline Fasting plasma glucose (mg/dL) & 93.1 & \pm & 9.7 & 91.7 & \pm & 8.7 & 92.7 & \pm & 9.5 & 91.8 & \pm & 10.3 & 0.153 \\
\hline Hemoglobin A1c (\%) & 5.4 & \pm & 0.4 & 5.4 & \pm & 0.4 & 5.4 & \pm & 0.4 & 5.4 & \pm & 0.3 & 0.294 \\
\hline Fasting insulin (IU/L) ${ }^{\mathrm{a}}$ & 4.7 & $(3.0$ & $-7.0)$ & 4.4 & $(3.0$ & & 4.9 & $(3.0$ & $-8.0)$ & 5.3 & $(3.0$ & $-7.8)$ & 0.004 \\
\hline HOMA-IR ${ }^{\mathrm{a}}$ & 1.1 & $(0.7$ & $-1.6)$ & 1.0 & $(0.7$ & & 1.1 & $(0.8$ & $-1.7)$ & 1.2 & $(0.6$ & $-1.6)$ & 0.014 \\
\hline Total cholesterol (mg/dL) & 207.2 & \pm & 31.9 & 201.8 & \pm & 31.9 & 207.8 & \pm & 34.8 & 210.1 & \pm & 35.2 & 0.101 \\
\hline Triglycerides (mg/dL) ${ }^{\mathrm{a}}$ & 104 & $(68-$ & 151) & 93 & $(78-$ & & 105 & $(68-$ & 161) & 110 & $(66-$ & 148) & 0.086 \\
\hline HDL-cholesterol (mg/dL) & 59.1 & \pm & 15.4 & 58.3 & \pm & 13.7 & 57.8 & \pm & 14.6 & 56.5 & \pm & 13.6 & 0.037 \\
\hline Total energy intake (kcal/day) & 1998 & \pm & 535 & 2084 & \pm & 511 & 2257 & \pm & 573 & 2627 & \pm & 776 & $<0.001$ \\
\hline Total fiber intake (g/day) & 10.2 & \pm & 4.2 & 10.2 & \pm & 3.9 & 11.1 & \pm & 4.2 & 12.3 & \pm & 5.2 & $<0.001$ \\
\hline Total fiber intake (g/1,000kcal) & 5.1 & \pm & 1.5 & 4.9 & \pm & 1.5 & 4.9 & \pm & 1.4 & 4.6 & \pm & 1.2 & $<0.001$ \\
\hline $\begin{array}{l}\text { Sugar-sweetened beverage intake } \\
\text { (serving/day) }\end{array}$ & 0 & & & 0.12 & $(0.1$ & $-0.21)$ & 0.48 & $(0.3$ & $0-0.84)$ & 2.1 & (1.4 & $-2.7)$ & \\
\hline
\end{tabular}




\begin{tabular}{|c|c|c|c|c|c|c|c|c|c|}
\hline Diet soda intake (serving/day) & 0.00 & $(0.00-0.00)$ & 0.00 & $(0.00-0.00)$ & 0.00 & $(0.00-0.12)$ & 0.00 & $(0.00-0.30)$ & $<0.001$ \\
\hline 100\% fruit juice intake (serving/day) & 0.00 & $(0.00-0.00)$ & 0.00 & $(0.00-0.09)$ & 0.00 & $(0.00-0.23)$ & 0.00 & $(0.00-0.23)$ & $<0.001$ \\
\hline Vegetable juice intake (serving/day) & 0.00 & $(0.00-0.00)$ & 0.00 & $(0.00-0.09)$ & 0.00 & $(0.00-0.09)$ & 0.00 & $(0.00-0.00)$ & 0.063 \\
\hline Coffee intake (serving/day) & 1.10 & $(0.40-1.50)$ & 1.50 & $(0.40-1.50)$ & 1.50 & $(0.40-1.50)$ & 1.00 & $(0.25-1.90)$ & 0.054 \\
\hline Family history of diabetes (\%) & 13.0 & & 16.6 & & 12.7 & & 14.1 & & 0.414 \\
\hline Smoking status (\%) & & & & & & & & & 0.326 \\
\hline Non-smoker & 30.3 & & 34.7 & & 30.9 & & 36.5 & & \\
\hline Ex-smoker & 15.9 & & 11.4 & & 14.8 & & 14.9 & & \\
\hline Current smoker & 53.8 & & 53.9 & & 54.3 & & 48.5 & & \\
\hline Alcohol drinking (\%) & & & & & & & & & $<0.001$ \\
\hline Never & 11.1 & & 14.4 & & 16.1 & & 24.5 & & \\
\hline Occasional & 8.6 & & 11.4 & & 11.3 & & 12.4 & & \\
\hline Drink $<20$ g/day & 33.8 & & 31.7 & & 31.3 & & 27.0 & & \\
\hline Drink $\geq 20$ g/day & 46.5 & & 42.4 & & 41.3 & & 36.1 & & \\
\hline Habitual exercise - Yes (\%) & 29.1 & & 26.2 & & 27.1 & & 29.5 & & 0.692 \\
\hline Prevalence - BMI $\geq 25 \mathrm{~kg} / \mathrm{m}^{2}$ (\%) & 25.0 & & 20.7 & & 29.0 & & 34.4 & & 0.006 \\
\hline Prevalence - Hypertension (\%) & 36.8 & & 33.2 & & 35.0 & & 32.4 & & 0.557 \\
\hline Prevalence - Dyslipidemia (\%) & 33.8 & & 28.4 & & 32.6 & & 32.8 & & 0.462 \\
\hline Dietary intervention- Yes (\%) & 7.9 & & 8.1 & & 7.9 & & 7.5 & & 0.995 \\
\hline
\end{tabular}

Data are presented as $n$, mean \pm standard deviation, median (interquartile range), or $\%$. 
${ }^{\mathrm{a}}$ Data are geometric means (interquartile range). Log-transformed values were used for the analyses.

${ }^{\mathrm{b}} P$-values for linear trends for continuous variables and chi-squared test for categorical variables. The $P$-values for linear trends were calculated with the continuous variables based on ordinal variables containing the median value of diet soda consumption for each drink category. 
Table 2. Baseline characteristics of the 2,037 participants according to diet-soda consumption category

\begin{tabular}{|c|c|c|c|c|c|c|c|c|c|c|c|c|c|}
\hline & \multicolumn{13}{|c|}{ Diet soda consumption } \\
\hline & \multicolumn{3}{|c|}{ Rare or never } & \multicolumn{3}{|c|}{$\begin{array}{c}\text { > rare/never } \\
\text { but }<1 \text { serving/week }\end{array}$} & \multicolumn{3}{|c|}{$\begin{array}{l}\geq 1 \text { serving/week } \\
\text { to }<1 \text { serving/day }\end{array}$} & \multicolumn{3}{|c|}{$\geq 1$ serving/day } & \multirow[t]{2}{*}{$\mathrm{p}^{\mathrm{b}}$} \\
\hline $\mathrm{N}$ & 1620 & & & 198 & & & 199 & & & 20 & & & \\
\hline Age (years) & 46.4 & \pm & 6.0 & 46.3 & \pm & 5.9 & 44.9 & \pm & 6.0 & 42.3 & \pm & 5.2 & $<0.001$ \\
\hline BMI $\left(\mathrm{kg} / \mathrm{m}^{2}\right)$ & 23.3 & \pm & 2.9 & 23.7 & \pm & 2.9 & 23.7 & \pm & 2.9 & 24.9 & \pm & 2.6 & 0.003 \\
\hline Systolic blood pressure (mmHg) & 120.5 & \pm & 18.5 & 118.6 & \pm & 19.3 & 122.3 & \pm & 14.5 & 121.1 & \pm & 13.5 & 0.636 \\
\hline Diastolic blood pressure (mmHg) & 77.7 & \pm & 13.1 & 75.7 & \pm & 13.2 & 79.4 & \pm & 10.9 & 79.6 & \pm & 11.5 & 0.313 \\
\hline Fasting plasma glucose (mg/dL) & 92.7 & \pm & 9.5 & 92.0 & \pm & 8.9 & 92.9 & \pm & 10.3 & 88.5 & \pm & 11.8 & 0.076 \\
\hline Hemoglobin A1c (\%) & 5.4 & \pm & 0.4 & 5.4 & \pm & 0.3 & 5.4 & \pm & 0.4 & 5.4 & \pm & 0.3 & 0.862 \\
\hline Fasting insulin (IU/L) ${ }^{\mathrm{a}}$ & 4.8 & $(3.0$ & $-7.0)$ & 5.0 & $(3.0$ & & 5.1 & $(3.0$ & $3.0)$ & 4.8 & (3.0 & $-7.8)$ & 0.644 \\
\hline HOMA-IR ${ }^{\mathrm{a}}$ & 1.1 & $(0.7$ & $-1.6)$ & 1.2 & $(0.7$ & & 1.2 & $(0.8$ & l.7) & 1.0 & $(0.6$ & $-1.6)$ & 0.946 \\
\hline Total cholesterol (mg/dL) & 206.9 & \pm & 33.9 & 206.6 & \pm & 32.7 & 208.1 & \pm & 32.1 & 214.3 & \pm & 36.4 & 0.300 \\
\hline Triglycerides (mg/dL) ${ }^{\mathrm{a}}$ & 102 & $(68$ & 151) & 112 & $(78-$ & & 107 & $(68-$ & 61) & 98 & $(66-$ & -148) & 0.961 \\
\hline HDL-cholesterol (mg/dL) & 58.3 & \pm & 14.8 & 56.9 & \pm & 12.9 & 57.4 & \pm & 13.9 & 61.1 & \pm & 20.0 & 0.624 \\
\hline Total energy intake (kcal/day) & 2155 & \pm & 582 & 2267 & \pm & 639 & 2375 & \pm & 727 & 2849 & \pm & 829 & $<0.001$ \\
\hline Total fiber intake (g/day) & 10.6 & \pm & 4.2 & 11.3 & \pm & 4.5 & 11.9 & \pm & 5.2 & 13.2 & \pm & 5.1 & $<0.001$ \\
\hline Total fiber intake (g/1,000kcal) & 4.9 & \pm & 1.4 & 5.0 & \pm & 1.3 & 5.0 & \pm & 1.5 & 4.7 & \pm & 1.2 & 0.579 \\
\hline $\begin{array}{l}\text { Sugar-sweetened beverage } \\
\text { consumption (serving/day) }\end{array}$ & 0.12 & $(0.0$ & $0-0.45)$ & 0.30 & $(0.1$ & $0.60)$ & 0.30 & $(0.53$ & $-0.93)$ & 1.9 & $(1.1$ & $-2.7)$ & $<0.001$ \\
\hline
\end{tabular}




\begin{tabular}{|c|c|c|c|c|c|c|c|c|c|}
\hline Diet soda consumption (serving/day) & 0 & & 0.12 & $(0.12-0.12)$ & 0.30 & $(0.30-0.60)$ & 2.6 & $(2.1-3.2)$ & \\
\hline $\begin{array}{l}\text { 100\% fruit juice consumption } \\
\text { (serving/day) }\end{array}$ & 0.00 & $(0.00-0.09)$ & 0.09 & $(0.00-0.23)$ & 0.09 & $(0.00-0.23)$ & 0.10 & $(0.00-0.71)$ & $<0.001$ \\
\hline $\begin{array}{l}\text { Vegetable juice intake consumption } \\
\text { (serving/day) }\end{array}$ & 0.00 & $(0.00-0.00)$ & 0.00 & $(0.00-0.09)$ & 0.00 & $(0.00-0.18)$ & 0.00 & $(0.00-0.39)$ & 0.518 \\
\hline Coffee consumption (serving/day) & 1.50 & $(0.40-1.50)$ & 1.05 & $(0.40-1.50)$ & 0.70 & $(0.40-1.50)$ & 0.65 & $(0.20-2.25)$ & 0.712 \\
\hline Family history of diabetes (\%) & 13.5 & & 13.2 & & 14.6 & & 10.0 & & 0.936 \\
\hline Smoking status (\%) & & & & & & & & & 0.740 \\
\hline Non-smoker & 31.5 & & 33.3 & & 32.2 & & 45.0 & & \\
\hline Ex-smoker & 14.8 & & 12.6 & & 17.1 & & 10.0 & & \\
\hline Current smoker & 53.8 & & 54.0 & & 50.8 & & 45.0 & & \\
\hline Alcohol drinking (\%) & & & & & & & & & 0.178 \\
\hline Never & 14.7 & & 14.1 & & 20.6 & & 15.0 & & \\
\hline Occasional & 9.8 & & 13.6 & & 13.6 & & 15.0 & & \\
\hline Drink $<20$ g/day & 31.7 & & 31.8 & & 31.2 & & 30.0 & & \\
\hline Drink $\geq 20 \mathrm{~g}$ /day & 43.8 & & 40.4 & & 34.7 & & 40.0 & & \\
\hline Habitual exercise - Yes (\%) & 27.0 & & 32.8 & & 30.2 & & 25.0 & & 0.311 \\
\hline Prevalence - BMI $\geq 25 \mathrm{~kg} / \mathrm{m}^{2}(\%)$ & 26.2 & & 29.8 & & 31.7 & & 40.0 & & 0.055 \\
\hline Prevalence - Hypertension (\%) & 34.6 & & 33.3 & & 38.7 & & 50.0 & & 0.320 \\
\hline Prevalence - Dyslipidemia (\%) & 32.1 & & 34.8 & & 32.2 & & 40.0 & & 0.768 \\
\hline Dietary intervention- Yes (\%) & 7.1 & & 10.1 & & 11.1 & & 15.0 & & 0.076 \\
\hline
\end{tabular}

Data are presented as $n$, mean \pm standard deviation, median (interquartile range), or $\%$. 
${ }^{a}$ Data are geometric means (interquartile range). Log-transformed values were used for the analyses.

${ }^{\mathrm{b}} P$-values for linear trends for continuous variables and chi-squared test for categorical variables. The $P$-values for linear trends were calculated with the continuous variables based on ordinal variables containing the median value of diet soda consumption for each drink category. 
Table 3. Risk of incident type 2 diabetes according to soft drink and diet soda consumption categories in 2,037 Japanese men

\begin{tabular}{cccc}
\hline & Consumption of soft drinks \\
\cline { 2 - 4 } & Rare or never & rare/never but & $\geq 1$ serving/week \\
$<1$ serving/week & to $<1$ serving/day
\end{tabular}$\quad$\begin{tabular}{c} 
P for trend serving/day $^{\text {a }}$ \\
\hline
\end{tabular}

\section{Sugar-sweetened beverage}

n

Number of incident cases

Person-years of follow-up

Incidence rate (/1,000 person-years)

Hazard ratio (95\% confidence interval)

Model 1

Model 4

\section{Diet soda}

n

Number of incident cases

Person-years of follow-up

Incidence rate (/1,000 person-years)

Hazard ratio (95\% confidence interval)

$\begin{array}{lcccc}660 & 271 & 865 & 241 & \\ 55 & 19 & 72 & 24 & \\ 3554 & 1494 & 4825 & 1381 & \\ 15.5 & 12.7 & 14.9 & 17.4 & \\ & & & & \\ & & & & \\ 0 \text { (reference) } & 0.86(0.51-1.45) & 1.03(0.72-1.46) & 1.24(0.77-2.01) & 0.296 \\ 0 \text { (reference) } & 0.92(0.55-1.56) & 0.99(0.70-1.41) & 1.20(0.74-1.94) & 0.389 \\ 0 \text { (reference) } & 0.90(0.80-2.27) & 1.06(0.74-1.53) & 1.35(0.80-2.27) & 0.208 \\ 0 \text { (reference) } & 0.97(0.57-1.64) & 1.11(0.74-1.66) & 1.34(0.72-2.36) & 0.424\end{array}$

1620

198

199

20

124

8968

16

1066

28

2

1103

116

13.8

15.0

25.4

17.2 


\begin{tabular}{lllll} 
Model 1 & 1.00 (reference) & $1.10(0.66-1.86)$ & $1.99(1.33-2.98)$ & 0.001 \\
Model 2 & 1.00 (reference) & $1.05(0.62-1.76)$ & $1.82(1.22-2.71)$ & 0.005 \\
Model 3 & 1.00 (reference) & $1.05(0.62-1.78)$ & $1.70(1.13-2.55)$ & 0.013 \\
Model 4 & 1.00 (reference) & $1.14(0.66-1.95)$ & $1.71(1.11-2.63)$ & 0.015 \\
\hline
\end{tabular}

Model 1: adjusted for age; Model 2, adjusted for age and BMI; Model 3, adjusted for the variables in Model 2 above + family history of diabetes, smoking, alcohol drinking, and habitual exercise, presence of hypertension, presence of dyslipidemia, receiving the diet treatment for chronic disease, total energy intake, and total fiber intake; Model 4, adjusted for the variables in Model 3 above + consumption of sugar-sweetened beverage (for diet soda), diet soda consumption (for sugar-sweetened beverage), fruit juice consumption, vegetable juice consumption, and coffee consumption.

The Cox proportional hazard model was used for the analyses.

${ }^{a}$ Linear regression was used for continuous variables based on ordinal variables containing the median value for each drink category. 